\title{
Pacific
}

Journal of

Mathematics

\section{DEGREE-ONE MAPS ONTO LENS SPACES}

Claude Hayat-Legrand, Shicheng Wang and Heiner Zieschang 


\section{DEGREE-ONE MAPS ONTO LENS SPACES}

\section{Claude Hayat-Legrand, Shicheng Wang and Heiner Zieschang}

The paper deals with the question about the existence or non-existence of a degree-one map of a closed orientable 3manifold $M$ to some lens space. The answer to this question is determined by the cyclic decomposition of $H_{1}(M)$, except when $H_{1}(M)$ contains an even number of direct factors isomorphic to $Z_{2^{k}}$. In this case one has to calculate the linking matrix of $M$ to get the answer. For every $n$ even, we give a Seifert manifold $M_{n}$ with $H_{1}\left(M_{n}\right) \cong \mathbf{Z}_{n} \oplus \mathbf{Z}_{n}$ that does not admit a degree-one map to $L(n, m)$ for any $m$.

\section{Introduction.}

Motivated by articles by Y. Rong [10] also Y. Rong and S. Wang [11], we consider the question whether a given 3-manifold $M$ admits a degree-one map to a lens space $L(n, m)$ or not. For every $n$ a geometric criterium is given (Theorem 2.2) by the linking pairing Tor $H_{1}(M) \otimes$ Tor $H_{1}(M) \rightarrow$ $\mathbf{Q} / \mathbf{Z},(\alpha, \beta) \mapsto \alpha \odot \beta$. Moreover it has the advantage of being quite simple and useful for the geometric construction of examples with a negative answer. Because it is a pairing on abelian group it is easy to prove (Theorem 2.10) that the answer to the question about the existence of a degree-one map to a $L(n, m)$ is determined by the cyclic decomposition of $H_{1}(M)$ except for the case where $n=2^{k} \bar{n}, k>0, \bar{n}$ odd and $H_{1}(M)$ contains an even number of direct factors isomorphic to $\mathbf{Z}_{2^{k}}$. In the end one has to calculate the linking pairing only in this case.

For the case $L(n, m)=L(2,1)=\mathbf{P}^{3}$ the often used method depends on the existence of a generator $\xi \in H^{1}\left(L(2,1), \mathbf{Z}_{2}\right)$ with the property $\xi \cup \xi \cup \xi \neq$ 0 . If $n$ is odd or a multiple of 4 (see for example Remark 2.8 (c)) there is no element $\xi \in H^{1}\left(L(n, m), \mathbf{Z}_{n}\right)$ with $\xi^{3} \neq 0$, but there is a generator $\mu \in H_{1}(L(n, m))$ with linking number $\mu \odot \mu \neq 0[\mathbf{1 6}, 14.7 .3$ (c)]. This is the ingredient for Theorem 2.2. If there is a degree-one map $M \rightarrow L(n, m)$ then $H_{1}(M)$ has a direct factor isomorphic to $\mathbf{Z}_{n}$, see [2], [16, 14.2.6]. Exploring the geometric description of the linking number we construct for every even $n$ a Seifert manifold $M_{n}$ with $H_{1}\left(M_{n}\right)=\mathbf{Z}_{n} \oplus \mathbf{Z}_{n}$ such that there is no degreeone map from $M_{n}$ to a lens space $L(n, m)$. If $n=2$ the Seifert manifold $M_{2}$ 
does not contain an embedded closed non-orientable surface of odd Euler characteristic (Theorem 4.1).

Degree-one maps to $\mathbf{P}^{3}$ appear in another context, namely in the theory of kinks which are related to Riemannian 4-manifolds with a Lorentz metric. The homotopy classification of Lorentz metrics on a space-time model reduces to the study of the homotopy classes $\left[M^{3}, \mathbf{P}^{3}\right]$ where $M^{3}$ corresponds to the space part. In $[\mathbf{1 3}]$ it is shown that $\left[M^{3}, \mathbf{P}^{3}\right]$ is an abelian group isomorphic to $\mathbf{Z} \oplus \mathbf{Z}_{2}^{\ell}$. The number of kinks (i.e. the number of tiping over of the light cone) appears in the factor $\mathbf{Z}$ and depends on the existence or non-existence of a degree-one map $M^{3} \rightarrow \mathbf{P}^{3}$. In [13] one asks to determine the existence or non-existence of degree-one maps to $\mathbf{P}^{3}$ for all closed connected orientable 3-manifolds. The theory of kinks has recently again been considered by G. W. Gibbons and S. W. Hawking [4] in the more general theory when the topology of the space changes during the time.

Discussions with Ralph Stöcker have been of great influence to this paper. Jeff Williams and Peter Zvengrowski introduced us to the theory of kinks and Daryl Cooper suggested Theorem 4.1. We thank the referee for his help, in particular for the better formulation of Theorem 2.10.

\section{A criterion for the existence of degree-one maps.}

Notations 2.1. For lens spaces we use the usual notation $L(n, m)$ and understand at all times that $n>1$ and $\operatorname{gcd}(n, m)=1$.

In the following we use the group $\mathbf{Q} / \mathbf{Z}$. An element of this group is denoted by $\left[\frac{r}{s}\right]$ or, mostly, by $[r / s]$ where $r, s \in \mathbf{Z}, s \neq 0, \operatorname{gcd}(r, s)=1$; this stands for the coset $\frac{r}{s}+\mathbf{Z}$. Given $[r / s]$ we denote by $\left[r^{-1} / s\right]$ the class $\left[r^{\prime} / s\right]$ with $r r^{\prime} \equiv 1 \bmod s$.

For a closed connected oriented 3-manifold $N$ we use the following homomorphisms:

$-j_{n}: H^{1}\left(N, \mathbf{Z}_{n}\right) \rightarrow H^{1}(N, \mathbf{Q} / \mathbf{Z})$ induced by the monomorphism $j: \mathbf{Z}_{n} \rightarrow$ $\mathbf{Q} / \mathbf{Z}, x+n \mathbf{Z} \mapsto[x / n] ;$

- the Bockstein operator $B: H^{1}(N, \mathbf{Q} / \mathbf{Z}) \rightarrow H^{2}(N)$, see [16, 14.7.4];

- the Bockstein operator $B_{n}=\mu_{n} \circ B \circ j_{n}: H^{1}\left(N, \mathbf{Z}_{n}\right) \rightarrow H^{2}\left(N, \mathbf{Z}_{n}\right)$ where $\mu_{n}$ is induced by the projection $\mu: \mathbf{Z} \rightarrow \mathbf{Z}_{n}$;

- the Poincaré duality isomorphism $D: H^{2}(N) \rightarrow H_{1}(N)$ defined by $D(\gamma)=\gamma \cap\{N\}$ where $\{N\}$ is the fundamental class of $N$.

For any $\xi, \eta \in$ Tor $H_{1}(N)$, we denote by $\xi \odot \eta$ the linking number of $\xi$ and $\eta$. This is an element of $\mathbf{Q} / \mathbf{Z}$. (For the definition and notation see $[16,14.7 .2]$.) 
Theorem 2.2. Let $M$ be a closed connected orientable 3-manifold.

(a) Assume that there is an element $\alpha \in H_{1}(M)$ of order $n>1$ such that the linking number $\alpha \odot \alpha$ is equal to $[r / n]$ where $r$ is prime to $n$. Then there exists a degree-one map $f: M \rightarrow L(n, s)$ where $s$ is the inverse of $r$ modulo $n$.

(b) If there is a degree-one map $f: M \rightarrow L(n, m)$ then there exists an element $\alpha \in$ Tor $H_{1}(M)$ such that $\alpha \odot \alpha=\left[m^{-1} / n\right]$, the order of $\alpha$ is $n$ and $\alpha$ generates a direct factor of $H_{1}(M)$.

Lemma 2.3. Let $f: M \rightarrow L(n, m)$ be a continuous mapping of degree $d$. For $\ell \in \mathbf{Z}$ there is a mapping $M \rightarrow L(n, m)$ of degree $d+\ell n$.

Proof. Let $g: S^{3} \rightarrow S^{3}$ be of degree $\ell$ and $\pi: S^{3} \rightarrow L(n, m)$ be the universal covering. Then $\tilde{g}=\pi \circ g: S^{3} \rightarrow L(n, m)$ is of degree $\ell n$. Now the composition of maps

$$
M \stackrel{\approx}{\longrightarrow} M \# S^{3} \longrightarrow M \vee S^{3} \stackrel{f \vee \tilde{g}}{\longrightarrow} L(n, m)
$$

gives the desired mapping of degree $d+\ell n$.

Proposition 2.4. If $\alpha$ and $\alpha \odot \alpha$ have the same finite order $n$ then $\alpha$ is a generator of a direct factor of $H_{1}(M)$.

Proof. Otherwise there is an element $\beta \in H_{1}(M)$ such that $\alpha=q \beta$ and the orders of $\beta$ and $q$ have a common divisor:

$$
\begin{aligned}
& \operatorname{order}(\beta)=m^{k} b, \operatorname{gcd}(m, b)=1, q=m^{\ell} q_{0}, \operatorname{gcd}\left(m, q_{0}\right)=1, \\
& \operatorname{order}(\alpha)=m^{k-\ell} a, a \mid b, 0<\ell<k .
\end{aligned}
$$

Since the order of $\beta \odot \beta$ divides the order of $\beta$ we have $m^{k} b=\operatorname{order}(\beta \odot \beta) \cdot s$ and $\beta \odot \beta=\left[\frac{s t}{m^{k} b}\right]$ for suitable $s$ and $t$. Now, for some $r$ relatively prime to $m^{k-\ell} a$

$$
\left[\frac{r}{m^{k-\ell} a}\right]=\alpha \odot \alpha=q^{2} \beta \odot \beta=\left[q_{0}^{2} \frac{s t m^{\ell}}{m^{k-\ell} b}\right] \text {. }
$$

Thus

$$
b r \equiv a q_{0}^{2} s t m^{\ell} \quad \bmod m^{k-\ell} a b,
$$

contradicting $\operatorname{gcd}(m, b r)=1$.

Taking another generator of the group generated by $\alpha$ changes the $r$ by multiplying it by a square and Corollary 2.5 follows:

Corollary 2.5. If Tor $H_{1}(M)=\mathbf{Z}_{n} \oplus A$ where $\operatorname{gcd}(n,|A|)=1$ then the homotopy type of $L(n, m)$ in Theorem 2.2 is uniquely determined.

Proof of 2.2. Consider the lens space $L(n, s)$ where $s r \equiv 1 \bmod n$; in the following we will write $s=r^{-1}$ and $L=L(n, s)$. 
First we recall that the mapping

$$
[M, L] \rightarrow \operatorname{Hom}\left(H_{1}(M), H_{1}(L)\right), \quad f \mapsto f_{*}
$$

is surjective. This is obtained as follows. By the universal coefficient theorem, there is an isomorphism $H^{1}\left(M, H_{1}(L)\right)=\operatorname{Hom}\left(H_{1}(M), H_{1}(L)\right)$. Thus each element $f_{*} \in \operatorname{Hom}\left(H_{1}(M), H_{1}(L)\right)$ determines a homotopy class, represented by $\hat{f}: M \rightarrow K\left(\pi_{1}(L), 1\right)$. Remember that $K\left(\pi_{1}(L), 1\right)$ can be constructed with $L$ as 3-skeleton; let $\rho: L \hookrightarrow K\left(\pi_{1}(L), 1\right)$ be the embedding. By the cellular approximation theorem we may assume that $\hat{f}(M) \subset L$. This gives a map $f: M \rightarrow L$ such that $\rho \circ f=\hat{f}$. The result can also be obtained using standard obstruction arguments.

By $[16,15.6 .3]$, there is an $\hat{\alpha} \in H^{1}\left(M, \mathbf{Z}_{n}\right)$ such that

$$
j\left\langle\hat{\alpha} \cup B_{n} \hat{\alpha},\{M\}\right\rangle=\alpha \odot \alpha=\left[\frac{r}{n}\right] .
$$

From $[\mathbf{1 6}, 14.7 .3$ (c), 15.6.3] it follows for the lens space $L=L(n, s)$ that there are generators $\mu \in H_{1}\left(L, \mathbf{Z}_{n}\right)$ and $\hat{\mu} \in H^{1}\left(L, \mathbf{Z}_{n}\right)$ with

$$
\mu \odot \mu=j\left\langle\hat{\mu} \cup B_{n} \hat{\mu},\{L\}\right\rangle=\left[\frac{r}{n}\right] .
$$

Next we prove that the $f: M \rightarrow L$ above can be chosen such that $f^{*}(\hat{\mu})=$ $\hat{\alpha}$. To see this, define

$$
\begin{aligned}
\varphi: H_{1}(M) \rightarrow \mathbf{Z}_{n}, & \varphi(a)=\langle\hat{\alpha}, a\rangle, \\
\psi: H_{1}(L) \rightarrow \mathbf{Z}_{n}, & \psi(b)=\langle\hat{\mu}, b\rangle .
\end{aligned}
$$

Since $\hat{\mu}$ is a generator of $H^{1}\left(L, \mathbf{Z}_{n}\right)$ the homomorphism $\psi$ is an isomorphism and the homomorphism

$$
\psi^{-1} \circ \varphi: H_{1}(M) \rightarrow H_{1}(L)
$$

is well defined. By (1) there is an $f: M \rightarrow L$ with $f_{*}=\psi^{-1} \circ \varphi$; hence, $\psi \circ f_{*}=\varphi$. Now, for any $a \in H_{1}(M)$,

$$
\langle\hat{\alpha}, a\rangle=\varphi(a)=\psi f_{*}(a)=\left\langle\hat{\mu}, f_{*} a\right\rangle=\left\langle f^{*} \hat{\mu}, a\right\rangle
$$

and this implies $\hat{\alpha}=f^{*}(\hat{\mu})$ by the universal coefficient theorem.

Let $d$ be the degree of $f$. Then

$$
\begin{aligned}
r+n \mathbf{Z} & =\left\langle\hat{\alpha} \cup B_{n} \hat{\alpha},\{M\}\right\rangle=\left\langle f^{*} \hat{\mu} \cup B_{n} f^{*} \hat{\mu},\{M\}\right\rangle \\
& =\left\langle\hat{\mu} \cup B_{n} \hat{\mu}, f_{*}\{M\}\right\rangle=\left\langle\hat{\mu} \cup B_{n} \hat{\mu}, d\{L\}\right\rangle=d \cdot(r+n \mathbf{Z}) .
\end{aligned}
$$


This shows that $d \equiv 1 \bmod n$. Now Lemma 2.3 gives the existence of a mapping $M \rightarrow L(n, s)$ of degree 1 .

For the proof of the second assertion of Theorem 2.2, let $f: M \rightarrow L(n, m)$ be a degree-one map. As mentioned at the beginning of the proof, there exists a generator $\mu$ of $H_{1}(L(n, m)) \cong \mathbf{Z}_{n}$ such that $\mu \odot \mu=\left[m^{-1} / n\right]$. Define $\alpha \in$ Tor $H_{1}(M)$ by $\alpha=D B j_{n} f^{*}(\hat{\mu})$. Then the order of $\alpha$ divides $n$ and $\alpha \odot \alpha=\mu \odot \mu=\left[m^{-1} / n\right] \neq 0$ see $[16,15.6 .3]$; hence $\operatorname{order}(\alpha)=n$. By Proposition 2.4, $\alpha$ generates a direct factor of $H_{1}(M)$.

Remark 2.6. Every $\alpha \in H^{1}\left(M, \mathbf{Z}_{n}\right)$ gives a homotopy class of maps $\bar{\alpha}: M \rightarrow K\left(\mathbf{Z}_{n}, 1\right)$. It is possible to consider $K\left(\mathbf{Z}_{n}, 1\right)$ as the infinite lens space $L_{n}^{\infty}$ with some $L(n, m)$ (usual 3-dimensional lens space) as 3-skeleton. Because the dimension of $M$ is 3 and $\pi_{2}(L(n, m))$ is 0 , there is no obstruction to lifting $\bar{\alpha}$ to obtain a map $f: M \rightarrow L(n, m)$. Now the problem is: does there exist an $m$ such that $f$ is a degree-one map? When $n$ is prime proceeding geometrically like Greenberg and Harper [4, page 295], or using $H^{3}\left(L(n, m), \mathbf{Z}_{n}\right)=H^{3}\left(K\left(\mathbf{Z}_{n}, 1\right), \mathbf{Z}_{n}\right)$ and the well known cohomology of the Eilenberg-MacLane spaces, it is possible to find an element $\hat{\mu} \in H^{3}\left(L(n, m), \mathbf{Z}_{n}\right)$ such that $\hat{\mu} \cup B_{n}(\hat{\mu}) \neq 0$. The criterium may be obtained taking the corresponding element in the cohomology of $M$. If this element is not 0 it determines $m$. In this case the criterion is an easy generalization of Edmonds' result [3].

Y. Rong and S. Wang [11, Cor. 6] gave another proof of the following corollary.

Corollary 2.7. $\quad$ There exists a degree-one map $f: L(p, q) \rightarrow L(n, m)$ if and only if $p=k n$ and $m \equiv k q c^{2} \bmod n$ where $k$ and $c$ are integers. In particular, there is a degree-one map $f: L(p, q) \rightarrow \mathbf{P}^{3} \approx L(2,1)$ if and only if $p \equiv 2 \bmod 4$.

Proof. Since $L(p, q) \approx L\left(p, q^{-1}\right)$ we may replace $f: L(p, q) \rightarrow L(n, m)$ by $f: L\left(p, q^{-1}\right) \rightarrow L\left(n, m^{-1}\right)$. Choose $\alpha \in H_{1}\left(L\left(p, q^{-1}\right)\right)$ with the property that $\alpha \odot \alpha=[q / p]$. Then each generator of $H_{1}\left(L\left(p, q^{-1}\right)\right)$ is of the form $c \alpha$ with $\operatorname{gcd}(p, c)=1$.

Suppose that $f: L\left(p, q^{-1}\right) \rightarrow L\left(n, m^{-1}\right)$ is a degree-one map. By 2.2 (b), there is $\beta \in H_{1}\left(L\left(p, q^{-1}\right)\right)$ of order $n$ such that $\beta \odot \beta=[m / n]$; hence, $p=k n, k \in \mathbf{Z}$, and $\beta=k c \alpha$ for some $c \in \mathbf{Z}$. Then

$\left[\frac{m}{n}\right]=\beta \odot \beta=k c \alpha \odot k c \alpha=\left[\frac{k^{2} c^{2} q}{p}\right]=\left[\frac{k c^{2} q}{n}\right] \Longrightarrow m \equiv k q c^{2} \quad \bmod n$.

The other direction follows from 2.2 (a) using the same calculation. 


\section{Remarks 2.8.}

(a) Since for $\mathbf{Z}_{2}$-coefficients the Bockstein operator $B_{2}$ satifies $B_{2}(\hat{\alpha})=$ $\hat{\alpha} \cup \hat{\alpha}$ it follows that

$$
\frac{1}{2}\langle\hat{\alpha} \cup \hat{\alpha} \cup \hat{\alpha},\{M\}\rangle=\alpha \odot \alpha \quad \text { where } \alpha=D B \hat{\alpha} .
$$

Hence, to determine the existence of a degree-one map $M \rightarrow \mathbf{P}^{3}$, the criteria $\xi^{3} \neq 0, \xi \in H^{1}\left(M, \mathbf{Z}_{2}\right)$ from [13], [17] and $\alpha \odot \alpha \neq 0, \alpha \in$ Tor $H_{1}(M)$ from 2.2 are equivalent.

(b) In the criterion of $[\mathbf{1 3}]$ the condition $\hat{\alpha} \cup \hat{\alpha} \cup \hat{\alpha} \neq 0$ cannot be changed to $\hat{\alpha} \cup \hat{\alpha} \neq 0$ as the following example shows. Let $N(K)$ be the twisted orientable $I$-bundle over the Klein bottle. Glue two copies together to give $M=$ $N(K) \cup_{T} N(K), T=\partial N(K)$ such that $H_{1}(M)=\mathbf{Z}_{2} \oplus \mathbf{Z}_{2}$. (Attention: $M$ is not the double of $N(K)$.) Then $H^{1}\left(M, \mathbf{Z}_{2}\right)=\mathbf{Z}_{2} \oplus \mathbf{Z}_{2}$ where the Poincaré duals of the generators of the two factors are the two Klein bottles central in the two copies of $N(K)$. If $\hat{\alpha} \in H^{1}\left(M, \mathbf{Z}_{2}\right)$ is one of these generators then $\hat{\alpha} \cup \hat{\alpha} \neq 0$ but $\hat{\alpha} \cup \hat{\alpha} \cup \hat{\alpha}=0$, see Fig. 1 for the intersection of the duals.

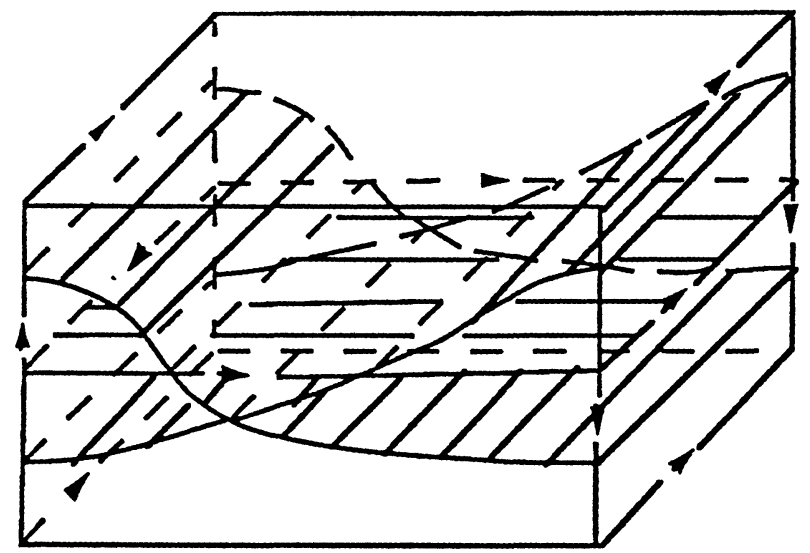

Figure 1.

(c) For a lens space $L=L(n, m), n$ even, there is a convenient geometric way to calculate $\hat{\alpha} \cup \hat{\alpha} \cup \hat{\alpha}$ for $\hat{\alpha} \in H^{1}\left(L, \mathbf{Z}_{n}\right)=\mathbf{Z}_{n}$. This is a special case of the calculation in [8, Lemma 3.4] where the arguments are used for arbitrary manifolds. Consider the Heegaard splitting $L=V_{1} \cup V_{2}$ of genus 1 and let $c_{i}$ be the core of $V_{i}$. Each $c_{i}$ defines a generator of $H_{1}(L) \cong \mathbf{Z}_{n}$ and $c_{2} \sim m c_{1}$. Let $\hat{\alpha}$ be the Hom-dual of $c_{1}$. Taking the meridian disk $D_{1} \subset V_{1}$ 
and extending it by an annulus parallel to $\partial D_{1}$ in $V_{2}$ and by the core $c_{2}$ we obtain a singular surface $F_{1}$ such that $\partial F_{1}=n c_{2},\left|F_{1} \cap c_{1}\right|=1$. Then the homology class defined by $F_{1}$ is the Poincaré dual of $\hat{\alpha}$. Let $F_{1}^{\prime}$ be a parallel copy of $F_{1}$ where the singular curve is a curve in $V_{2}$ parallel but disjoint to $c_{2}$. Furthermore we take a singular surface $F_{2}$ with the roles of the two solid tori interchanged. Then $D_{2} \cap\left(F_{1} \cup F_{1}^{\prime}\right) \subset V_{2}$ consists of two points with stars of $n$ segments which intersect as shown in the figure. The number of intersection points of these stars equals :

$$
2\left(1+2+\ldots+\left(\frac{n}{2}-1\right)\right)+\frac{n}{2}=\left(\frac{n}{2}\right)^{2} .
$$

See Fig. 2. Hence $\hat{\alpha} \cup \hat{\alpha} \cup \hat{\alpha}=m^{-1}\left(\frac{n}{2}\right)^{2}\{\hat{L}\} \in H^{3}\left(L, \mathbf{Z}_{n}\right)=\mathbf{Z}_{n}$ and this is different from 0 if and only if $n \equiv 2 \bmod 4$.

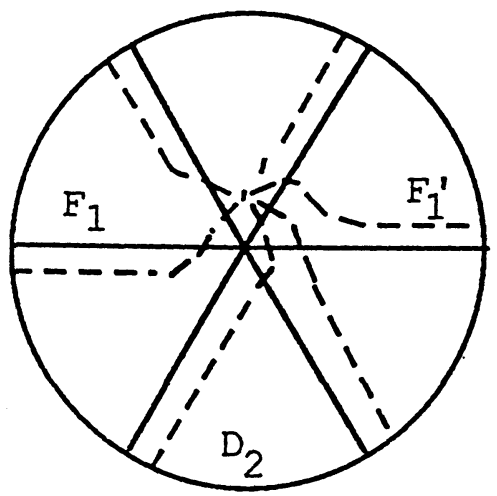

Figure 2.

For the next theorem we need the following lemma.

Lemma 2.9. Let $M$ be a closed orientable 3-manifold and let $n_{1}, n_{2}$ be two coprime integers. If there are degree-one maps $M \rightarrow L\left(n_{1}, m_{1}\right)$ and $M \rightarrow L\left(n_{2}, m_{2}\right)$ then there is a degree-one map $M \rightarrow L\left(n_{1} n_{2}, m\right)$.

Proof. By Theorem 2.2, there exist

$\alpha_{i} \in H_{1}(M) \quad$ with $\quad n_{i} \alpha_{i}=0, \alpha_{i} \odot \alpha_{i}=\left[r_{i} / n_{i}\right], r_{i} m_{i} \equiv 1 \quad \bmod n_{i}, i=1,2$. 
Let $\alpha=n_{2} \alpha_{1}+n_{1} \alpha_{2}$. Then

$$
\begin{aligned}
n_{1} n_{2} \alpha & =0 \\
\alpha \odot \alpha & \equiv n_{2}^{2} \alpha_{1} \odot \alpha_{1}+2 n_{1} n_{2} \alpha_{1} \odot \alpha_{2}+n_{1}^{2} \alpha_{2} \odot \alpha_{2} \quad \bmod \mathbf{Z} \\
& \equiv n_{2}^{2}\left[r_{1} / n_{1}\right]+n_{1}^{2}\left[r_{2} / n_{2}\right] \quad \bmod \mathbf{Z} \\
& \equiv\left[r / n_{1} n_{2}\right] \quad \bmod \mathbf{Z}
\end{aligned}
$$

where $r=n_{2}^{3} r_{1}+n_{1}^{3} r_{2}$ is prime to $n_{1} n_{2}$. By $2.2(\mathrm{a})$, there is a degree-one map $M \rightarrow L(n, m)$ where $m r \equiv 1 \bmod n$.

Theorem 2.10. Let $M$ be an orientable closed 3-manifold.

(a) If there is a degree one map $f: M \rightarrow L(n, m)$, then $H_{1}(M)=\mathbf{Z}_{n} \oplus A$.

(b) If $H_{1}(M)=\mathbf{Z}_{n} \oplus A$ where $n>1$ is odd, then there is a degree one map $f: M \rightarrow L(n, m)$ for some $m$ with $\operatorname{gcd}(n, m)=1$.

(c) If $H_{1}(M)=\mathbf{Z}_{n} \oplus A$ where $n=2^{k} \bar{n}$, for some $k>0$ and $\bar{n}$ odd, then there exists a degree-one map $f: M \rightarrow L(n, m)$ for some $m, \operatorname{gcd}(n, m)$ $=1$ provided $A$ contains an even number of factors $\mathbf{Z}_{2^{k}}$.

The additional assumption on $A$ in (c) is necessary, as examples in the next section will show.

Proof. (a) The result follows from Theorem 2.2 (b).

(b) By Lemma 2.9, it is enough to consider the case where $n=p^{k}$, where $p$ is an odd prime. By [18, Theorem (4)], see also [6, page 29], there is a $\alpha \in$ Tor $H_{1}(M)$ such that $\alpha \odot \alpha=\left[r(p) / p^{k}\right]$ with $\operatorname{gcd}(r(p), p)=1$. The conclusion follows from Theorem 2.2 (a).

(c) Since $\mathbf{Z}_{n}=\mathbf{Z}_{2^{k}} \oplus \mathbf{Z}_{\bar{n}}$, by Lemma 2.9 , it is enough to show that there is a degree-one map $f: M \rightarrow L\left(2^{k}, m\right)$ for some odd $m$. By [18, Theorem (4)], there is a basis of $H_{1}(M)$ that contains an odd number of generators of order $2^{k}$ such that parts of the matrix of the linking pairing for these generators consist of diagonal blocks of the form

$$
\left[m / 2^{k}\right],\left(\begin{array}{cc}
0 & {\left[1 / 2^{k}\right]} \\
{\left[1 / 2^{k}\right]} & 0
\end{array}\right) \quad \text { or } \quad\left(\begin{array}{c}
{\left[1 / 2^{k-1}\right]\left[1 / 2^{k}\right]} \\
{\left[1 / 2^{k}\right]\left[1 / 2^{k-1}\right]}
\end{array}\right)
$$

Hence, for at least one of the generators the self-linking number is $\left[m / 2^{k}\right] \neq 0$, and now the assertion follows from Theorem 2.2.

\section{Remarks 2.11.}

(a) Parts of the above results have been known. For $H_{1}(M)=\mathbf{Z}_{n}$ LuftSjerve [7] gave an answer by proving the existence of a special surface. Using 
the argument of [7], E. Luft and Y. Rong independently observed that there exists a degree-one map $f: M \rightarrow L(n, m)$ if $H_{1}(M)=\mathbf{Z}_{n} \oplus A$ and the order of Tor $A$ is relatively prime to $n$. This is a consequence of 2.10 .

(b) For many Seifert fiber spaces $M$ one can easily prove the existence of a degree-one map onto some lens space $L\left(2^{k}, m\right)$, proceeding from the canonical presentation of the fundamental group of $M$ to the homology of $M$ with $\mathbf{Z}_{2}$ coefficients (see below). But this approach does not give negative answers to the existence of degree-one maps onto a fixed lens space or any lens space.

\section{On the non-existence of degree-one maps.}

Construction 3.1. In the following we denote curves, their homotopy and homology classes by the same symbol. Consider the Seifert manifold $M=M\left(\varepsilon_{1} / n_{1}, \varepsilon_{2} / n_{2}, \varepsilon_{3} / n_{3}\right), \varepsilon_{i}= \pm 1$ obtained from $\left(S^{2}-\stackrel{\circ}{D_{1}} \cup \stackrel{\circ}{D_{2}} \cup \stackrel{\circ}{D_{3}}\right) \times$ $S^{1}$ by attaching the union of three solid tori $T_{1} \cup T_{2} \cup T_{3}$ with a glueing map described below; here $\stackrel{\circ}{D}_{1}, \ldots$ are open discs and $T_{1}, \ldots$ fibered solid tori. Denote $\partial D_{i}$ by $x_{i}$ and the fiber $S^{1}$ by $h$. The meridian of the fibered solid torus $T_{i}$ is denoted by $a_{i}$ and its longitude by $b_{i}$. The glueing map is defined, up to isotopy, by the relations $n_{i} x_{i}+\varepsilon_{i} h=a_{i}$ and $x_{i}=b_{i}$ (such that the determinant is \pm 1 ). Then

$$
\begin{aligned}
& \pi_{1}\left(M\left(\varepsilon_{1} / n_{1}, \varepsilon_{2} / n_{2}, \varepsilon_{3} / n_{3}\right)\right) \\
& =\left\langle x_{1}, x_{2}, x_{3}, h \mid x_{i}^{n_{i}} h^{\varepsilon_{i}}=1,1 \leq i \leq 3, x_{1} x_{2} x_{3}=1\right\rangle .
\end{aligned}
$$

Notice that these relations imply that $h$ is in the center of $\pi_{1}(M)$.

Theorem 3.2. For $n$ even the Seifert manifold $M=M(-1 / n,-1 / n, 1 / n)$ admits no degree-one map to a lens space $L(n, m)$ ( $m$ arbitrary) and for $n=2^{k}$ to any lens space, but $H_{1}(M)=\mathbf{Z}_{n} \oplus \mathbf{Z}_{n}$.

Proof. Abelianizing $\pi_{1}(M)$ gives

$$
\begin{aligned}
H_{1}(M) & =\left\langle x_{1}, x_{2} \mid n x_{1}=n x_{2}=0\right\rangle^{a b}=\mathbf{Z}_{n} \oplus \mathbf{Z}_{n} \\
& =\langle\xi, \eta \mid n \xi=n \eta=0\rangle^{a b} \quad \text { where } \xi=x_{1}, \quad \eta=x_{1}-x_{2} .
\end{aligned}
$$

(For $n=2$ the order of $\pi_{1}(M)$ equals 8 , for $n>2$ the order is infinite.) To calculate $\eta \odot \eta$, we have to find a singular surface $F_{1,2}$ such that $\partial F_{1,2}=n \eta$. It is possible to describe this surface in three parts. One part lies in $T_{2}$ and is obtained from an annulus by identifying one boundary component with the curve $h=-a_{2}+n b_{2}$ on $\partial T_{2}$ while the other boundary component runs $n$ times along the core $-b_{2}$ of $T_{2}$. (Here $b_{2}$ denotes both the longitude and 
the core of the solid torus which are homotopic in $T_{2}$.) Another part is a singular annulus in $T_{1}$ with one boundary component running $n$ times along $b_{1}$ and the other being the curve $h=-a_{1}+n b_{1}$ on $\partial T_{1}$. These two parts are connected by an annulus $I \times h$. Then

$$
\partial F_{1,2}=n b_{1}-n b_{2}=n\left(x_{1}-x_{2}\right)=n \eta .
$$

Now we have to calculate the intersection number of $F_{1,2}$ with $x_{2}$ and $x_{1}$. We recall that $x_{2} \subset\left(S^{2}-\stackrel{\circ}{D}_{1} \cup \stackrel{\circ}{D}_{2} \cup \stackrel{\circ}{D}_{3}\right) \times S^{1}$ is the boundary of the open disc $\stackrel{\circ}{D}_{2}$ and that it cuts once the curve $-a_{2}+n b_{2}$ on $\partial T_{2}$ which is homologous to $h$. The same argument applies to the intersection of $x_{1}$ with $F_{1,2}$. Therefore

$$
\eta \odot x_{2}=[ \pm 1 / n], \eta \odot x_{1}=[ \pm 1 / n] \quad \text { so } \quad \eta \odot \eta=[ \pm 1 / n- \pm 1 / n] .
$$

By the same construction as above we find a singular surface $F_{2,3}$ with

$$
\partial F_{2,3}=n b_{2}+n b_{3}=n\left(x_{2}+x_{3}\right)=-n x_{1}=-n \xi .
$$

Since $F_{2,3}$ intersects $\partial T_{3}$ on $-h$ and $h$ intersects $x_{3}$ once, we obtain $\xi \odot x_{3}=$ $[ \pm 1 / n] ;$ similarly the intersection of $F_{2,3}$ and $\partial T_{2}$ is $h$ and $\xi \odot x_{2}=[ \pm 1 / n]$. Therefore

$$
\xi \odot \xi=\xi \odot x_{1}=\xi \odot\left(-x_{3}-x_{2}\right)=-\xi \odot x_{3}-\xi \odot x_{2}=[\mp 1 / n+\mp 1 / n] .
$$

For each element $\alpha \in H_{1}(M)=\mathbf{Z}_{n} \oplus \mathbf{Z}_{n}$ we have $\alpha=a \xi+b \eta$ and $\alpha \odot \alpha$ is of the form $[2 k / n]$. Therefore an equation $\alpha \odot \alpha=[r / n]$ with $\operatorname{gcd}(r, n)=1$ is impossible if $n$ is even. By Theorem 2.2, $M$ admits no degree-one map to $L(n, m)$ and hence, if $n=2^{k}$, to any lens space (since otherwise the homology group of this lens space is a free factor of $\left.H_{1}(M)\right)$.

Example 3.3.(due to A. Shastri and P. Zvengrowski.) Shastri-Zvengrowski [14] completely determined the existence of a degree-one map of any quotient of $S^{3}$ by a free action of a finite group to $\mathbf{P}^{3}$. The most difficult case was the manifold $M=S^{3} / Q_{4 n}$ where $Q_{4 n}$ is the generalized quaternion group $n \geq 1$. (The double cover $S^{3} \rightarrow S O(3)$ lifts the dihedral group with $2 n$ elements to a group $Q_{4 n}$ of order $4 n$.) It has the presentation

$$
Q_{4 n}=\left\langle x, y \mid x^{n}=y^{2}, x y x=y\right\rangle .
$$

For $n=2 m$ the homology groups do not suffice to solve the problem and Shastri-Zvengrowski used a free $\mathbf{Z} Q_{8 m}$-resolution of $\mathbf{Z}$ to calculate explicitly 
the ring structure of $H^{*}\left(K\left(Q_{8 m}, 1\right) ; \mathbf{Z}_{2}\right)$. We will show next that their result can also be obtained by our geometric method.

Consider the following presentation of $Q_{8 m}$.

$$
\begin{aligned}
\pi_{1}(M) & =\left\langle x, y, h \mid y^{2}=h, x^{2 m}=h, x y x=y\right\rangle \\
& =\left\langle x, y, z, h \mid y^{2}=h, x^{2 m}=h, z^{2}=h^{-1}, x y z=1\right\rangle . \\
& =\pi_{1}(M(-1 / 2 m,-1 / 2,1 / 2)) .
\end{aligned}
$$

Since the fundamental groups of the Seifert fiber spaces $M$ and $M(-1 / 2 m,-1 / 2,1 / 2)$ are isomorphic to $Q_{8 m}$ the spaces are homeomorphic, see $[9$, p. 110]. Clearly, $h$ is in the center of the group and a presentation of the abelian group $H_{1}(M)$ is as follows:

$$
\begin{aligned}
H_{1}(M) & =\langle x, y \mid 2(m x-y)=0,2 x=0\rangle^{a b} \\
& =\langle\xi, \eta \mid 2 \xi=0,2 \eta=0\rangle^{a b} \cong \mathbf{Z}_{2} \oplus \mathbf{Z}_{2}
\end{aligned}
$$

with $\xi=x$ and $\eta=m x-y$.

To calculate $\eta \odot \eta$ in the same manner as above we find a singular surface $F_{x y}$ such that $\partial F_{x y}=2 \eta$. Now we have to calculate the intersection number of $F_{x y}$ with $y$ and $x$ (there is no need to worry about the sign of the intersection numbers). We recall that $y \subset\left(S^{2}-\stackrel{\circ}{D}_{x} \cup \stackrel{\circ}{D}_{y} \cup \stackrel{\circ}{D}_{z}\right) \times S^{1}$ is the boundary of the open disc $\stackrel{\circ}{D}_{y}$ and that it cuts once the curve $-a_{y}+2 b_{y}$ on $\partial T_{y}$ which is homologous to $h$. The same argument applies to the intersection of $x$ with $F_{x y}$. Therefore

$$
\eta \odot y=[1 / 2], \eta \odot x=[1 / 2] \quad \text { so } \quad \eta \odot \eta=[(m-1) / 2] \text {. }
$$

We may apply the first part of Theorem 2.2 to get a degree-one map $S^{3} / Q_{8 m} \rightarrow L(2,1) \approx \mathbf{P}^{3}$ if $m$ is even.

Next we calculate $\xi \odot \xi$. Take a singular surface $F_{y z}$ (constructed in the same manner as above) such that $\partial F_{y z}=2 y-2 z$. Then we have $(y-z) \odot y=$ [1/2], $(y-z) \odot z=[1 / 2]$. From $z=-x-y$ we obtain $(2 y+x) \odot y=[1 / 2]$ and $(2 y+x) \odot(x+y)=[1 / 2]$. Substituting $x=\xi, y=-\eta+m \xi$ it follows from $2 \eta=0=2 \xi$ that

$$
-\xi \odot \eta+m \xi \odot \xi=[1 / 2],-\xi \odot \eta+(m+1) \xi \odot \xi=[1 / 2] .
$$

By adding these two equations we obtain $\xi \odot \xi=0$ and $\xi \odot \eta=[1 / 2]$. If $m$ is odd we have

$(\xi+\eta) \odot(\xi+\eta)=\xi \odot \xi+\xi \odot \eta+\eta \odot \xi+\eta \odot \eta=0+[1 / 2]+[1 / 2]+0=0$.

Since $\xi, \eta, \xi+\eta$ are the only non-zero elements of $H_{1}(M)=\mathbf{Z}_{2} \oplus \mathbf{Z}_{2}$ it follows from Theorem 2.2 (b) that the manifold $S^{3} / Q_{8 m}$ does not admit a degree-one map to a lens space if $m$ is odd. 


\section{Embedding closed surface with odd Euler caracteristic.}

By G. Bredon and J. Wood [1, page 88], the existence of an element of $H^{1}\left(M, \mathbf{Z}_{2}\right)$ whose cube-cup is not zero is equivalent to the existence of a closed non-orientable surface of odd Euler characteristic embedded in $M$. The proof in [1] is based on the theory of $L$-equivalence. Here we will give a geometric proof (similar to Rubinstein's proof [12, Theorem 18]) that the existence of a degree-one map $M \rightarrow \mathbf{P}^{3}$ is equivalent to the existence of an embedding of closed non-orientable surface of odd Euler characteristic to $M$.

Theorem 4.1. Let $M$ be a closed orientable 3-manifold. The following three assertions are equivalent:

(a) There exists a degree-one map $f: M \rightarrow \mathbf{P}^{3}$;

(b) There exists an embedded closed surface $F$ in $M$ with odd Euler characteristic;

(c) There exists $\zeta \in H^{1}\left(M ; \mathbf{Z}_{2}\right)$ with $\zeta \cup \zeta \cup \zeta \neq 0$.

Proof. For $(a) \Longleftrightarrow(c)$ see [13, Theorem 4.4] or Remark 2.8.

(b) $\Longrightarrow(a)$ : If the Euler characteristic of $F$ is odd then $F=F^{\prime} \# \mathbf{P}^{2}=$ $F_{0} \cup_{\partial F_{0}=\partial \mathcal{M}} \mathcal{M}$, with $F^{\prime}$ closed, orientable, $\stackrel{\circ}{D}$ an open disc, $F_{0}=F^{\prime}-\stackrel{\circ}{D}$, and $\mathcal{M}=\mathbf{P}^{2}-\stackrel{\circ}{D}$. The surface $\mathcal{M}$ is the Möbius band. Let $N(F)$ be a regular neighbourhood of $F$ in $M$. Then $N(F)$ is an $I$-bundle over $F$. Let $p: N(F) \rightarrow F$ be the projection of this bundle. The $I$-bundle $p^{-1}\left(F_{0}\right)$ is a product $F_{0} \times I$, otherwise there is an orientation reversing loop in $M$ (which is assumed orientable). The $I$-bundle $p^{-1}(\mathcal{M})$ is twisted (otherwise the center curve of $\mathcal{M}$ is an orientation reversing loop in $M$ ).

Let $N\left(\mathbf{P}^{2}\right)$ be a regular neighbourhood of $\mathbf{P}^{2}=D^{2} \cup_{\partial D^{2}=\partial \mathcal{M}} \mathcal{M}$ in $\mathbf{P}^{3}$ where $\mathcal{M}$ again is the Möbius band and $N(\mathcal{M})$ is the twisted $I$-bundle over $\mathcal{M}$, considered as part of $N\left(\mathbf{P}^{2}\right)$ :

$$
N\left(\mathbf{P}^{2}\right)=\left(D^{2} \times I\right) \cup N(\mathcal{M}) .
$$

Define $f: N(F) \rightarrow N\left(\mathbf{P}^{2}\right)$ as follows: $f \mid p^{-1}(\mathcal{M})$ sends $p^{-1}(\mathcal{M})$ to $N(\mathcal{M})$ homeomorphically and $f \mid p^{-1}\left(F_{0}\right)$ sends $p^{-1}\left(F_{0}\right)=F_{0} \times I$ to $D^{2} \times I$, inducing a proper degree-one map on the boundary. Now extend $f$ to a map $f:(M-N(F)) \rightarrow\left(\left(\mathbf{P}^{3}-N\left(\dot{P}^{2}\right)\right)=D^{3}\right)$ by mapping a collar of $\partial N(F)$ onto $D^{3}$ and the remaining part to the center point of $D^{3}$. The map $f$ is a degree-one map $M \rightarrow \mathbf{P}^{3}$.

$(a) \Longrightarrow(b)$ : Suppose $f: M \rightarrow \mathbf{P}^{3}$ is a degree-one map. Deforme $f$ to be transverse to $\mathbf{P}^{2} \subset \mathbf{P}^{3}$ such that $F=f^{-1}\left(\mathbf{P}^{2}\right)$ is connected. Let $N\left(\mathbf{P}^{2}\right)$ be a regular neighbourhood of $\mathbf{P}^{2}$ in $\mathbf{P}^{3}$. Then $\partial N\left(\mathbf{P}^{2}\right)$ is a 2-sphere. Now both 
maps $f \mid N(F): N(F) \rightarrow N\left(\mathbf{P}^{2}\right)$ and $f \mid \partial N(F): \partial N(F) \rightarrow \partial N\left(\mathbf{P}^{2}\right)$ are of degree 1. If $N(F)=F \times I$ then the degrees of the maps $f \mid F \times\{0\}: F \times\{0\} \rightarrow$ $\partial N\left(\mathbf{P}^{2}\right)$ and $f \mid F \times\{1\}: F \times\{1\} \rightarrow \partial N\left(\mathbf{P}^{2}\right)$ have the same absolute value; hence $f \mid \partial N(F): \partial N(F) \rightarrow \partial N\left(\mathbf{P}^{2}\right)$ is of even degree. Therefore $N(F)$ is a twisted $I$-bundle and, hence, $F$ is non-orientable.

Suppose the Euler characteristic of $F$ is even. Then $F=F^{\prime} \# K$ with $F^{\prime}$ closed orientable and $K$ a Klein bottle. Let $N(F)$ be a regular neighbourhood of $F$ in $M$. It is an $I$-bundle. This $I$-bundle over $F^{\prime}$ is a product because $M$ is orientable. The $I$-bundle over $K$ is twisted over every orientation reversing curve. Let $\alpha$ and $\beta$ represent the two generators of the fundamental group of $K$, where the loop $\alpha$ is two-sided and $\beta$ is one-sided. Then $\alpha$ is the only non-trivial element in Tor $H_{1}(F) \cong$ Tor $H_{1}(N(F))$ and, hence, the only candidate for $\alpha \odot \alpha \neq 0$. However one can push $F$ off itself in $N(F)$ so that the self-intersection of $F$ is $\alpha$. Since the $I$-bundle over $\alpha$ is trivial, $\alpha$ can be pushed off $F$. Then the intersection of $\alpha$ and $F$ is empty; thus $\alpha \odot \alpha=0$. (Compare Fig. 1.) By Remark 2.8 (a), this contradicts the existence of $\zeta \in H^{1}\left(M ; \mathbf{Z}_{2}\right)$ with $\zeta \cup \zeta \cup \zeta \neq 0$.

\section{References}

[1] G.E. Bredon and J.H. Wood, Non-orientable surfaces in orientable 3-manifolds, Inv. Math., 7 (1969), 83-110.

[2] W. Browder, Surgery on simply-connected manifolds, Springer-Verlag, Berlin-Heidelberg-New York, 1972.

[3] A. Edmonds, Construction of group actions on four manifolds, Trans. Amer. Math. Soc., 299 (1987), 155-170.

[4] G.W. Gibbons and S.W. Hawking, Kinks and topology change, Phys. Rev. Lett., 69 (1992), 1719-1721.

[5] M.J. Greenberg and J.R. Harper, Algebraic Topology, A First Course, Math. Lecture Note Series, Addison-Wesley Publishing Company, 1981.

[6] A. Kawauchi and S. Kojima, Algebraic classification of linking pairings on 3-manifolds, Math. Ann., 253 (1980), 29-42.

[7] E. Luft and D. Sjerve, Degree-1 maps onto lens spaces and free actions on homology spheres, Topology Appl., 37 (1990), 131-136.

[8] H. Murakami, T. Ohtsuki and M. Okada, Invariants of three-manifolds derived from linking matrices of framed links, Osaka J. Math., 29 (1992), 545-572.

[9] P. Orlik, Seifert Manifolds, Lecture Notes in Math., 291, Springer-Verlag, BerlinHeidelberg-New York, 1972.

[10] Y. Rong, Maps between Seifert fibered spaces of infinite $\pi_{1}$, Pacific J. Math., 160 (1993), 143-154.

[11] Y. Rong and S. Wang, The preimages of submanifolds, Math. Proc. Camb. Phil. Soc., 112 (1992), 271-279. 
[12] J.H. Rubinstein, One-sided Heegaard splittings of 3-manifolds, Pacific J. Math., 76 (1978), 185-200.

[13] A. Shastri, J.G. Williams and P. Zvengrowski, Kinks in general relativity, Internat. J. Theor. Phys., 19 (1980), 1-23.

[14] A. Shastri and P. Zvengrowski, Type of 3-manifolds and addition of relativistic kinks, Reviews in Math. Phys., 3 (1991), 467-478.

[15] E. Spanier, Algebraic Topology, McGraw-Hill Book Comp., New York, 1966.

[16] R. Stöcker and H. Zieschang, Algebraische Topologie, B.G. Teubner, Stuttgart, 1988.

[17] L.R. Taylor, Relative Rochlin invariants, Topology Appl., 18 (1984), 259-280.

[18] C.T.C. Wall, Quadratic forms on finite groups, and related topics, Topology, 2 (1964), 281-298.

Received February 15, 1994 and revised May 18, 1994.

LABORATOIRE DE TOPOLOGIE ET GÉOMÉtrie

U.R.A. ${ }^{\circ} 1408$, Université Paul Sabatier

UFRMIG, 118, ROUTE DE NARBONNE

31062 Toulouse-Cedex, France

E-mail address: hayat@Picard.ups-tlse.fr

AND

PeKING UNIVERSITY

BeIJING 100871, P.R. China

E-mail address: swang@sxx0.math.phu.edu.cn

AND

RUHR-UNIVERSITÄT BOCHUM

44780 Bochum, Germany

E-mail address: Marlene.Schwarz@rz.ruhr-uni-bochum.de 


\title{
PACIFIC JOURNAL OF MATHEMATICS
}

\author{
Founded in 1951 by
}

E. F. Beckenbach (1906-1982) $\quad$ F. Wolf (1904-1989)

\section{EDITORS}

Sun-Yung A. Chang (Managing Editor)

University of California

Los Angeles, CA 90095-1555

pacific@math.ucla.edu

F. Michael Christ

University of California

Los Angeles, CA 90095-1555

christ@math.ucla.edu

Nicholas Ercolani

University of Arizona

Tucson, AZ 85721

ercolani@math.arizona.edu
Robert Finn

Stanford University

Stanford, CA 94305

finn@gauss.stanford.edu

Steven Kerckhoff

Stanford University

Stanford, CA 94305

spk@gauss.stanford.edu

Martin Scharlemann

University of California

Santa Barbara, CA 93106

mgscharl@math.ucsb.edu
Gang Tian

Massachusettes Institute of Technology

Cambridge, MA 02139

tian@math.mit.edu

\author{
V. S. Varadarajan \\ University of California \\ Los Angeles, CA 90095-1555 \\ vsv@math.ucla.edu \\ Dan Voiculescu \\ University of California \\ Berkeley, CA 94720 \\ dvv@math.berkeley.edu
}

\section{SUPPORTING INSTITUTIONS}

ACADEMIA SINICA, TAIPEI

CALIF. INST. OF TECHNOLOGY

CHINESE UNIV. OF HONG KONG

HONG KONG UNIV. OF SCI. \& TECH.

KEIO UNIVERSITY

MACQUARIE UNIVERSITY

MATH. SCI. RESEARCH INSTITUTE

NEW MEXICO STATE UNIV.

OREGON STATE UNIV.

PEKING UNIVERSITY

RITSUMEIKAN UNIVERSITY

STANFORD UNIVERSITY

$\begin{array}{ll}\text { TOKYO INSTITUTE OF TECHNOLOGY } & \text { UNIV. OF CALIF., SANTA CRUZ } \\ \text { UNIVERSIDAD DE LOS ANDES } & \text { UNIV. OF HAWAII } \\ \text { UNIV. OF ARIZONA } & \text { UNIV. OF MELBOURNE } \\ \text { UNIV. OF BRITISH COLUMBIA } & \text { UNIV. OF MONTANA } \\ \text { UNIV. OF CALIF., BERKELEY } & \text { UNIV. NACIONAL AUTONOMA DE MEXICO } \\ \text { UNIV. OF CALIF., DAVIS } & \text { UNIV. OF NEVADA, RENO } \\ \text { UNIV. OF CALIF., IRVINE } & \text { UNIV. OF OREGON } \\ \text { UNIV. OF CALIF., LOS ANGELES } & \text { UNIV. OF SOUTHERN CALIFORNIA } \\ \text { UNIV. OF CALIF., RIVERSIDE } & \text { UNIV. OF UTAH } \\ \text { UNIV. OF CALIF., SAN DIEGO } & \text { UNIV. OF WASHINGTON } \\ \text { UNIV. OF CALIF., SANTA BARBARA } & \text { WASHINGTON STATE UNIVERSITY }\end{array}$

The supporting Institutions listed above contribute to the cost of publication of this Journal, but they are not owners or publishers and have no responsibility for its contents or policies.

\section{Manuscripts must be prepared in accordance with the instructions provided on the inside back cover.}

The table of contents and the abstracts of the papers in the current issue, as well as other information about the Pacific Journal of Mathematics, may be found on the Internet at http://www.math.uci.edu/pjm.html.

The Pacific Journal of Mathematics (ISSN 0030-8730) is published monthly except for July and August. Regular subscription rate: $\$ 245.00$ a year (10 issues). Special rate: $\$ 123.00$ a year to individual members of supporting institutions.

Subscriptions, back issues published within the last three years and changes of subscribers address should be sent to Pacific Journal of Mathematics, P.O. Box 4163, Berkeley, CA 94704-0163, U.S.A. Prior back issues are obtainable from Kraus Periodicals Co., Route 100, Millwood, NY 10546.

The Pacific Journal of Mathematics at the University of California, c/o Department of Mathematics, 981 Evans Hall, Berkeley, CA 94720 (ISSN 0030-8730) is published monthly except for July and August. Second-class postage paid at Berkeley, CA 94704, and additional mailing offices. POSTMASTER: send address changes to Pacific Journal of Mathematics, P.O. Box 6143, Berkeley, CA 94704-0163.

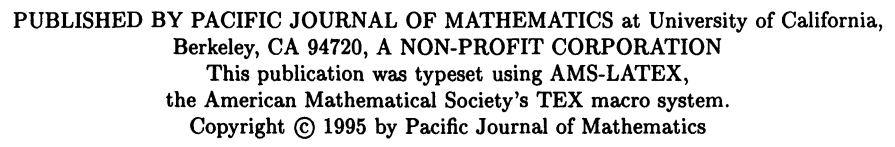




\title{
PACIFIC JOURNAL OF MATHEMATICS
}

\author{
Volume $176 \quad$ No. $1 \quad$ November 1996
}

Moduli spaces of isometric pluriharmonic immersions of Kähler manifolds into

indefinite Euclidean spaces

HitOSHI FURUHATA

On a theorem of Koch

FARSHID HAJIR

Degree-one maps onto lens spaces

Claude Hayat-Legrand, Shicheng WANG and Heiner Zieschang

Unitary representation induced from maximal parabolic subgroups for split $F_{4}$

CHENG CHON Hu

New constructions of models for link invariants

FRANÇOIS JAEGER

Solvability of Dirichlet problems for semilinear elliptic equations on certain domains

ZHIREN JIN

Hadamard-Frankel type theorems for manifolds with partially positive curvature

Katsuei KenMotsu and CHANGYU XIA

Boundary behavior of the Bergman curvature in strictly pseudoconvex polyhedral domains

KANG-TAE KIM and JIYE YU

Existence and behavior of the radial limits of a bounded capillary surface at a corner

KIRK LANCASTER and DAVID SIEGEL

Triangle subgroups of hyperbolic tetrahedral groups

COLIN MACLACHLAN

Chern classes of vector bundles on arithmetic varieties

TOHRU NAKASHIMA and YUICHIRO TAKEDA

Haar measure on $E_{q}(2)$

ARUP KUMAR PAL

Domains of partial attraction in noncommutative probability

VITTORINO PATA

Partitioning products of $\mathscr{P}(\omega) /$ fin

OTMAR SPINAS

Dimensions of nilpotent algebras over fields of prime characteristic

CORA M. STACK

Tensor products of structures with interpolation

FRIEDRICH WEHRUNG

Fourier multipliers for $L_{p}\left(\mathbb{R}^{n}\right)$ via $q$-variation 